\title{
MAXIMUM LIKELIHOOD DEGREE OF FERMAT HYPERSURFACES VIA EULER CHARACTERISTICS
}

\author{
BOTONG WANG \\ (Communicated by Lev Borisov)
}

\begin{abstract}
Maximum likelihood degree of a projective variety is the number of critical points of a general likelihood function. In this note, we compute the maximum likelihood degree of Fermat hypersurfaces. We give a formula of the maximum likelihood degree in terms of the constants $\beta_{\mu, \nu}$, which is defined to be the number of complex solutions to the system of equations $z_{1}^{\nu}=z_{2}^{\nu}=\cdots=z_{\mu}^{\nu}=1$ and $z_{1}+\cdots+z_{\mu}+1=0$.
\end{abstract}

\section{INTRODUCTION}

The maximum likelihood estimate is a fundamental problem in statistics. Maximum likelihood degree is the number of potential solutions to the maximum likelihood estimation problem on a projective variety. When the variety is smooth, Huh [H] showed that the maximum likelihood degree is indeed a topological invariant. If the variety is a general complete intersection, the maximum likelihood degree is computed in CHKS (see also [HS]).

In a recent preprint AAGL, Agostini, Alberelli, Grande and Lella studied the maximum likelihood degree of Fermat hypersurfaces. They obtained formulas for the maximum likelihood degree of a few special families of Fermat surfaces. However, their approach is through a case-by-case study.

In this note, we propose to compute the maximum likelihood degree of Fermat hypersurfaces in a more systematic way via a topological method. In general, the formula given in CHKS does not work for all the Fermat hypersurfaces, because the intersection of hypersurfaces

$$
\left\{x_{0}^{d}+x_{1}^{d}+\cdots+x_{n}^{d}=0\right\} \cap\left\{x_{0}+x_{1}+\cdots+x_{n}=0\right\} \subset \mathbb{P}^{n}
$$

may not be transverse. We will compute the error terms introduced by the nontransverse intersections. The main ingredient is Milnor's result on the topology of isolated hypersurfaces singularities. This topological approach is closely related to the approach of [BW] and [RW]. In fact, for an isolated hypersurface singularity, the Euler obstruction is up to a sign equal to the Milnor number plus one. So we essentially apply the ideas of [BW] and [RW] to these particular examples.

First, let us recall the definition of maximum likelihood degree. Let $\mathbb{P}^{n}$ be the $n$ dimensional complex projective space with homogeneous coordinates $\left(x_{0}, x_{1}, \ldots, x_{n}\right)$. Denote the coordinate plane $\left\{x_{i}=0\right\} \subset \mathbb{P}^{n}$ by $H_{i}$, and the hyperplane $\left\{x_{0}+x_{1}+\right.$ $\left.\cdots+x_{n}=0\right\}$ by $H_{+}$. Let the index set $\Lambda=\{0,1, \ldots, n,+\}$, and let $\mathcal{H}=\bigcup_{\lambda \in \Lambda} H_{\lambda}$.

Received by the editors September 24, 2015.

2010 Mathematics Subject Classification. Primary 14Q10; Secondary 32S50. 
Let $X \subset \mathbb{P}^{n}$ be a complex projective variety. Denote the smooth locus of $X$ by $X_{\text {reg. }}$. The maximum likelihood degree of $X$ is defined to be the number of critical points of the likelihood function

$$
l_{u}=\frac{x_{0}^{u_{0}} x_{1}^{u_{1}} \cdots x_{n}^{u_{n}}}{\left(x_{0}+x_{1}+\cdots+x_{n}\right)^{u_{0}+u_{1}+\cdots+u_{n}}}
$$

on $X_{\text {reg }} \backslash \mathcal{H}$ for generic $\left(u_{i}\right)_{0 \leq i \leq n} \in \mathbb{Z}^{n+1}$.

Theorem 1.1. Denote the Fermat hypersurface $\left\{x_{0}^{d}+x_{1}^{d}+\cdots+x_{n}^{d}=0\right\} \subset \mathbb{P}^{n}$ by $F_{n, d}$, and denote its maximum likelihood degree by $\operatorname{MLdeg}\left(F_{n, d}\right)$. Then,

$$
\operatorname{MLdeg}\left(F_{n, d}\right)=d+d^{2}+\cdots+d^{n}-\sum_{0 \leq j \leq n-1}\left(\begin{array}{c}
n+1 \\
j
\end{array}\right) \beta_{n-j, d-1}
$$

where $\beta_{\mu, \nu}$ is the number of complex solutions of the system of equations

$$
\begin{gathered}
z_{1}^{\nu}=z_{2}^{\nu}=\ldots=z_{\mu}^{\nu}=1, \\
z_{1}+\ldots+z_{\mu}+1=0 .
\end{gathered}
$$

When $\mu$ or $\nu$ is small, $\beta_{\mu, \nu}$ can be easily calculated. For example,

$$
\beta_{\mu, 1}=0 .
$$

$$
\beta_{1, \nu}= \begin{cases}0 & \text { if } \nu \text { is odd } \\ 1 & \text { if } \nu \text { is even }\end{cases}
$$

$$
\beta_{2, \nu}= \begin{cases}2 & \text { if } \nu \text { is divisible by } 3, \\ 0 & \text { otherwise. }\end{cases}
$$

With these calculations, we recover all the closed formulas in AAGL.

\section{Corollary 1.2.}

$$
\begin{gathered}
\operatorname{MLdeg}\left(F_{n, 2}\right)=2^{n+1}-2, \\
\operatorname{MLdeg}\left(F_{2, d}\right)= \begin{cases}d^{2}+d & \text { if } d \equiv 0,2 \bmod 6, \\
d^{2}+d-3 & \text { if } d \equiv 3,5 \bmod 6, \\
d^{2}+d-2 & \text { if } d \equiv 4 \bmod 6, \\
d^{2}+d-5 & \text { if } d \equiv 1 \bmod 6 .\end{cases}
\end{gathered}
$$

When $\nu$ is a power of a prime number, we have formulas to compute $\beta_{\mu, \nu}$. Equivalently, when $d-1$ is a power of a prime number, we have closed formulas for $\operatorname{MLdeg}\left(F_{n, d}\right)$. In fact, by a straightforward computation one can deduce the following corollary from Theorem 1.1 and Proposition 4.2 .

Corollary 1.3. Suppose $d-1=p^{r}$, where $p$ is a prime number and $r$ is a positive integer. Then

$\operatorname{MLdeg}\left(F_{n, d}\right)=d+d^{2}+\cdots+d^{n}-\frac{1}{d-1} \sum \frac{(n+1) !}{\left(n+1-p\left(s_{1}+\cdots+s_{k}\right)\right) ! \cdot\left(\left(s_{1}\right) ! \cdots\left(s_{k}\right) !\right)^{p}}$ where $k=\frac{d-1}{p}$ and the sum is over all nonnegative integers $s_{1}, \ldots, s_{k}$ with $1 \leq$ $s_{1}+\cdots+s_{k} \leq \frac{n+1}{p}$. 
To find a general formula for $\beta_{\mu, \nu}$ would be a very hard question in number theory and combinatorics. In fact, determining when $\beta_{\mu, \nu} \neq 0$ had been an open question for a long time, and it was solved by Lam and Leung [LL in 2000.

Since the Fermat hypersurface $F_{n, d}$ is smooth, by $[\underline{\mathrm{H}}] \operatorname{MLdeg}\left(F_{n, d}\right)$ is equal to the signed Euler characteristic $\chi\left(F_{n, d} \backslash \mathcal{H}\right)$. In section 2, we will compute $\chi\left(F_{n, d} \backslash \mathcal{H}\right)$, and we will postpone the technical calculation of the Milnor numbers to section 3 . In the last section, we will briefly discuss what we know about the constants $\beta_{n, d}$.

\section{Computing the Euler characteristics}

By the following theorem of Huh $[\mathrm{H}$, we reduce the problem of computing $\operatorname{MLdeg}\left(F_{n, d}\right)$ to computing $\chi\left(F_{n, d} \backslash \mathcal{H}\right)$. Recall that in $\mathbb{P}^{n}, \mathcal{H}=\bigcup_{\lambda \in \Lambda} H_{\lambda}$ is the union of all coordinate hyperplanes and the hyperplane $H_{+}=\left\{x_{0}+x_{1}+\cdots+x_{n}=\right.$ $0\}$.

Theorem 2.1 (Huh, [H]). If $X \subset \mathbb{P}^{n}$ is a subvariety such that $X \backslash \mathcal{H}$ is smooth, then

$$
\operatorname{MLdeg}(X)=(-1)^{\operatorname{dim}(X)} \chi(X \backslash \mathcal{H})
$$

Since the Euler characteristic is additive for algebraic varieties, by the inclusionexclusion principle,

$$
\chi(X \backslash \mathcal{H})=\sum_{0 \leq i \leq n} \sum_{\substack{\Lambda^{\prime} \subset \Lambda \\\left|\Lambda^{\prime}\right|=i}}(-1)^{i} \chi\left(X \cap H_{\Lambda^{\prime}}\right)
$$

where $H_{\Lambda^{\prime}}=\bigcap_{\lambda \in \Lambda^{\prime}} H_{\lambda}$.

The Fermat hypersurface $F_{n, d}=\left\{x_{0}^{d}+x_{1}^{d}+\cdots+x_{n}^{d}=0\right\}$ is invariant under any permutation of the coordinates. Therefore, (7) can be written as

$$
\chi\left(F_{n, d} \backslash \mathcal{H}\right)=\sum_{0 \leq i \leq n}(-1)^{i}\left(\left(\begin{array}{c}
n+1 \\
i
\end{array}\right) \chi\left(F_{n, d} \cap V^{i}\right)+\left(\begin{array}{c}
n+1 \\
i-1
\end{array}\right) \chi\left(F_{n, d} \cap W^{i}\right)\right)
$$

where $V^{i}=\bigcap_{0 \leq j \leq i-1} H_{j}$ and $W^{i}=H_{+} \cap \bigcap_{0 \leq j \leq i-2} H_{j}\left(W^{0}=\emptyset\right.$ and $\left.W^{1}=H_{+}\right)$.

$F_{n, d} \cap V^{i}$ is a smooth hypersurface in $\mathbb{P}^{n-i}$ of degree $d$. Euler characteristics of such hypersurfaces only depend on $n-i$ and $d$, and they are calculated in D, Chapter 5, (3.7). However, it turns out that we don't have to compute each of these Euler characteristics. For now, we simply denote the Euler characteristic of a smooth degree $d$ hypersurface in $\mathbb{P}^{m}$ by $e_{m, d}$. In particular,

$$
\chi\left(F_{n, d} \cap V^{i}\right)=e_{n-i, d} .
$$

$F_{n, d} \cap W^{i}$ is a possibly singular hypersurface in $W^{i}$ for $1 \leq i \leq n$. In fact, $F_{n, d} \cap W^{i}$ is isomorphic to the intersection of the Fermat hypersurface $F_{n-i+1, d} \subset$ $\mathbb{P}^{n-i+1}$ and the hyperplane $\left\{x_{0}+x_{1}+\cdots+x_{n-i+1}=0\right\}$. Using the Lagrange multiplier method, one can easily see that all the singular points of $F_{n, d} \cap W^{i}$ are isolated and there are exactly $\beta_{n-i+1, d-1}$ many of them. The Euler characteristics of such hypersurfaces can be computed using Milnor numbers. 
Theorem 2.2 ([D, Chapter $5(4.4)])$. For any singular point $P$ of $F_{n, d} \cap W^{i}$ we can define the Milnor number $\mu\left(F_{n, d} \cap W^{i}, P\right)$ by considering $F_{n, d} \cap W^{i}$ as a hypersurface of $W^{i}$. Then,

$$
\chi\left(F_{n, d} \cap W^{i}\right)=e_{n-i, d}+(-1)^{n-i} \sum_{P} \mu\left(F_{n, d} \cap W^{i}, P\right)
$$

where the sum is over all the singular points $P$ of $F_{n, d} \cap W^{i}$.

Proposition 2.3. For any singular point $P$ of $F_{n, d} \cap W^{i}$,

$$
\mu\left(F_{n, d} \cap W^{i}, P\right)=1 .
$$

We will postpone the proof of the proposition to the next section. The next corollary follows immediately from (10) and (11).

\section{Corollary 2.4.}

$$
\chi\left(F_{n, d} \cap W^{i}\right)=e_{n-i, d}+(-1)^{n-i} \beta_{n-i+1, d-1} .
$$

Now, combining (8), (9) and (12), we have

$$
\chi\left(F_{n, d} \backslash \mathcal{H}\right)=\sum_{0 \leq i \leq n}(-1)^{i}\left(\left(\begin{array}{c}
n+1 \\
i
\end{array}\right) e_{n-i, d}+\left(\begin{array}{c}
n+1 \\
i-1
\end{array}\right)\left(e_{n-i, d}+(-1)^{n-i} \beta_{n-i+1, d-1}\right)\right) .
$$

Since $\left(\begin{array}{c}n+1 \\ i\end{array}\right)+\left(\begin{array}{c}n+1 \\ i-1\end{array}\right)=\left(\begin{array}{c}n+2 \\ i\end{array}\right)$, (13) is equivalent to

(14) $\chi\left(F_{n, d} \backslash \mathcal{H}\right)=\sum_{0 \leq i \leq n}(-1)^{i}\left(\begin{array}{c}n+2 \\ i\end{array}\right) e_{n-i, d}+\sum_{1 \leq i \leq n}(-1)^{n}\left(\begin{array}{c}n+1 \\ i-1\end{array}\right) \beta_{n-i+1, d-1}$.

Suppose $X$ is a general hypersurface of degree $d$ in $\mathbb{P}^{n}$. Then (7) implies that

$$
\chi(X \backslash \mathcal{H})=\sum_{0 \leq i \leq n}(-1)^{i}\left(\begin{array}{c}
n+2 \\
i
\end{array}\right) e_{n-i, d} .
$$

The maximum likelihood degree of a general hypersurface is well understood.

Proposition 2.5 ([HS, 1.11]). The maximum likelihood of a general degree d hypersurface in $\mathbb{P}^{n}$ is equal to $d+d^{2}+\cdots+d^{n}$.

Combining the proposition, (15) and Theorem 2.1, we have

$$
\begin{aligned}
\sum_{0 \leq i \leq n}(-1)^{i}\left(\begin{array}{c}
n+2 \\
i
\end{array}\right) e_{n-i, d} & =\chi(X \backslash \mathcal{H}) \\
& =(-1)^{n-1} \operatorname{MLdeg}(X) \\
& =(-1)^{n-1}\left(d+d^{2}+\cdots+d^{n}\right) .
\end{aligned}
$$

Therefore, (14) is equivalent to

(17) $\chi\left(F_{n, d} \backslash \mathcal{H}\right)=(-1)^{n-1}\left(d+d^{2}+\cdots+d^{n}\right)+\sum_{1 \leq i \leq n}(-1)^{n}\left(\begin{array}{c}n+1 \\ i-1\end{array}\right) \beta_{n-i+1, d-1}$.

Again, by Theorem 2.1, we have

$$
\operatorname{MLdeg}\left(F_{n, d}\right)=d+d^{2}+\cdots+d^{n}-\sum_{1 \leq i \leq n}\left(\begin{array}{c}
n+1 \\
i-1
\end{array}\right) \beta_{n-i+1, d-1}
$$

which is the statement of Theorem 1.1 


\section{The Milnor numbers}

We prove Proposition 2.3 in this section.

For the geometric meaning of Milnor number, we refer to [D, Chapter 3]. Here we compute the Milnor numbers using Jacobian ideals. Denote the ring of germs of holomorphic functions at $0 \in \mathbb{C}^{l}$ by $\mathcal{O}$. Let $f \in \mathcal{O}$ be a nonzero germ of holomorphic function such that the germ of hypersurface $f^{-1}(0)$ has an isolated singularity at the origin $0 \in \mathbb{C}^{l}$. The Jacobian ideal of $f$, denoted by $J_{f}$ is defined by

$$
J_{f}=\left(\frac{\partial f}{\partial z_{1}}, \cdots, \frac{\partial f}{\partial z_{l}}\right) \subset \mathcal{O}
$$

where $z_{1}, \ldots, z_{l}$ are the coordinates of $\mathbb{C}^{n}$.

Theorem 3.1 ([D, Chapter 3, (2.7)]). The Milnor number of $f^{-1}(0)$ at the origin, denoted by $\mu\left(f^{-1}(0), 0\right)$, is given by the formula

$$
\mu\left(f^{-1}(0), 0\right)=\operatorname{dim}_{\mathbb{C}} \mathcal{O} / J_{f} .
$$

Recall that $W^{i}=\left\{x_{0}=x_{1}=\cdots=x_{i-2}=x_{0}+x_{1}+\cdots+x_{n}=0\right\} \subset \mathbb{P}^{n}$. Denote $y_{j}=x_{i-1+j}, 0 \leq j \leq n-i+1$. Then the intersection $F_{n, d} \cap W^{i}$ is isomorphic to the intersection

$$
\left\{y_{0}^{d}+y_{1}^{d}+\cdots+y_{n-i+1}^{d}=0\right\} \cap\left\{y_{0}+y_{1}+\cdots+y_{n-i+1}=0\right\}
$$

in $\mathbb{P}^{n-i+1}$. Without lost of generality, we can work on the affine space $y_{0} \neq 0$, and rewrite the intersection in affine coordinates

$$
\left\{1+\bar{y}_{1}^{d}+\cdots+\bar{y}_{n-i+1}^{d}=0\right\} \cap\left\{1+\bar{y}_{1}+\cdots+\bar{y}_{n-i+1}=0\right\} .
$$

Here we use $\bar{y}_{j}$ to denote the corresponding affine coordinate of $y_{j}$, that is, $\bar{y}_{j}=$ $y_{j} / y_{0}$. Suppose $\left(\xi_{1}, \ldots, \xi_{n-i+1}\right)$ is a singular point of the above intersection. Then by the Lagrange multiplier method,

$$
\xi_{1}^{d-1}=\xi_{2}^{d-1}=\cdots=\xi_{n-i+1}^{d-1}=1 .
$$

We can eliminate $\bar{y}_{n-i+1}$ by $\bar{y}_{n-i+1}=1-\bar{y}_{1}-\cdots-\bar{y}_{n-i}$. On this affine chart, $F_{n, d} \cap W^{i}$ is isomorphic to the hypersurface $\{f=0\}$ in $\mathbb{C}^{n-i}$, where

$$
f=1+\bar{y}_{1}^{d}+\cdots+\bar{y}_{n-i}^{d}+\left(1-\bar{y}_{1}-\cdots-\bar{y}_{n-i}\right)^{d} .
$$

Let $z_{j}=\bar{y}_{j}-\xi_{j}$. Then

$$
f=1+\left(z_{1}+\xi_{1}\right)^{d}+\cdots+\left(z_{n-i}+\xi_{n-i}\right)^{d}+\left(\xi_{n-i+1}-z_{1}-\cdots-z_{n-i}\right)^{d} .
$$

Proposition 3.2. In the local ring $\mathcal{O}$, the Jacobian ideal $J_{f}=\left(\frac{\partial f}{\partial z_{1}}, \ldots, \frac{\partial f}{\partial z_{n-i}}\right)$ is equal to the maximal ideal $\left(z_{1}, z_{2}, \ldots, z_{n-i}\right)$.

Proof. Notice that $\xi_{j}^{d-1}=1$ for all $1 \leq j \leq n-i+1$. Therefore,

$$
\begin{aligned}
\frac{\partial f}{\partial z_{j}} & =\frac{d(d-1)}{2} \cdot \xi_{j}^{d-2} z_{j}+\frac{d(d-1)}{2} \cdot \xi_{j}^{d-2}\left(z_{1}+\cdots+z_{n-i}\right)+\text { higher degree terms } \\
& =\frac{d(d-1)}{2} \cdot \xi_{j}^{d-2}\left(z_{1}+\cdots+z_{j-1}+2 z_{j}+z_{j+1}+\cdots+z_{n-i}\right)+\text { higher degree terms. }
\end{aligned}
$$

By Nakayama's lemma, we only need to show that the vectors $z_{1}+\cdots+z_{j-1}+$ $2 z_{j}+z_{j+1}+\cdots+z_{n-i}, 1 \leq j \leq n-i$ span the whole vector space $\mathbb{C} z_{1} \oplus \mathbb{C} z_{2} \oplus \cdots \oplus$ $\mathbb{C} z_{n-j}$. By adding all such vectors together, we see $z_{1}+z_{2}+\cdots+z_{n-i}$ is contained 
in their span. Thus

$$
z_{j}=\left(z_{1}+\cdots+z_{j-1}+2 z_{j}+z_{j+1}+\cdots+z_{n-i}\right)-\left(z_{1}+z_{2}+\cdots+z_{n-i}\right)
$$

is in the span.

Now, Proposition 2.3 follows from Theorem 3.1 and Proposition 3.2 .

\section{The COnstants $\beta_{\mu, \nu}$}

Instead of working with the constants $\beta_{\mu, \nu}$, we define $\alpha_{\mu, \nu}$ to be the number of complex solutions to the system of equations

$$
\left\{\begin{array}{l}
z_{1}^{\nu}=z_{2}^{\nu}=\cdots=z_{\mu}^{\nu}=1, \\
z_{1}+z_{2}+\cdots+z_{\mu}=0 .
\end{array}\right.
$$

Then clearly $\beta_{\mu, \nu}=\frac{1}{\nu} \cdot \alpha_{\mu+1, \nu}$. The advantage of working with $\alpha_{\mu, \nu}$ is that their defining equations have better symmetry.

We would like to answer the following question.

Question. Can we give a formula for $\alpha_{\mu, \nu}$ in terms of $\mu$ and the prime factorization of $\nu$ ?

This is definitely a very hard question. The work of Lam and Leung gives a necessary and sufficient condition of $\alpha_{\mu, \nu} \neq 0$.

Theorem 4.1 ([LL] $)$. Suppose $\nu=p_{1}^{a_{1}} \cdots p_{l}^{a_{l}}$ is the prime factorization. Then $\alpha_{\mu, \nu} \neq 0$ if and only if $\mu \in \mathbb{Z}_{\geq 0} \cdot p_{1}+\cdots+\mathbb{Z}_{\geq 0} \cdot p_{l}$.

When $\nu=p^{r}$ has only one prime factor, we can give a formula of $\alpha_{\mu, \nu}$. In this case, suppose $\left(z_{1}, \ldots, z_{\mu}\right)$ is a solution to (23). Then the collection $\left\{z_{1}, \ldots, z_{\mu}\right\}$ can be divided into groups of $p$ elements such that each group is a rotation of $1, e^{2 \pi i / p}, \ldots, e^{2(p-1) \pi i / p}$. Therefore, if $p$ does not divide $\mu$, then $\alpha_{\mu, \nu}=0$. If $p$ divides $\mu$, then

$$
\alpha_{\mu, \nu}=\sum \frac{\mu !}{\left(\left(s_{1}\right) !\left(s_{2}\right) ! \cdots\left(s_{k}\right) !\right)^{p}}
$$

where $k=\nu / p$, and the sum is over all $s_{1}, \ldots, s_{k} \in \mathbb{Z}_{\geq 0}$ such that $s_{1}+\cdots+s_{k}=\mu / p$. Since $\beta_{\mu, \nu}=\frac{1}{\nu} \cdot \alpha_{\mu+1, \nu}$, we can translate (24) into a statement about $\beta_{\mu, \nu}$.

Proposition 4.2. Suppose $\nu=p^{r}$, where $p$ is a prime number and $r$ is a positive integer. Then $\beta_{\mu, \nu}=0$ when $p$ does not divide $\mu+1$, and when $p$ divides $\mu+1$

$$
\beta_{\mu, \nu}=\frac{1}{\nu} \sum \frac{(\mu+1) !}{\left(\left(s_{1}\right) !\left(s_{2}\right) ! \cdots\left(s_{k}\right) !\right)^{p}}
$$

where $k=\nu / p$, and the sum is over all $s_{1}, \ldots, s_{k} \in \mathbb{Z}_{\geq 0}$ such that $s_{1}+\cdots+s_{k}=\frac{\mu+1}{p}$.

Suppose $\nu=p^{r} q^{s}$ has two distinct prime factors, and suppose $\left(z_{1}, \ldots, z_{\mu}\right)$ is a solution to (23). Then by [LL, Corollary 3.4], the collection $\left\{z_{1}, \ldots, z_{\mu}\right\}$ can be divided into groups of $p$ or $q$ elements such that each group is a rotation of $1, e^{2 \pi i / p}, \ldots, e^{2(p-1) \pi i / p}$ or a rotation of $1, e^{2 \pi / q}, \ldots, e^{2(q-1) \pi i / q}$ respectively. However, this decomposition is not unique, and this is the main difficulty to finding a formula for $\alpha_{\mu, \nu}$ in this case. Now, this is already a problem beyond our capability.

When $\nu$ has at least three distinct prime factors, the statement of LL, Corollary 3.4] is no longer true. Therefore, the question becomes much harder and deeper. 


\section{ACKNOWLEDGEMENT}

The author thanks Jiu-Kang Yu and Zhengpeng Wu for helpful discussions about the constants $\beta_{\mu, \nu}$.

\section{REFERENCES}

[AAGL] D. Agostini, D. Alberelli, F. Grande, and P Lella, The maximum likelihood degree of Fermat hypersurfaces, arXiv:1404.5745. To appear in J. Algebr. Stat.

[BW] N. Budur and B. Wang, Bounding the maximum likelihood degree, arXiv:1411.3486. To appear in Math. Res. Lett.

[CHKS] Fabrizio Catanese, Serkan Hoşten, Amit Khetan, and Bernd Sturmfels, The maximum likelihood degree, Amer. J. Math. 128 (2006), no. 3, 671-697. MR2230921(2007m:13036)

[D] Alexandru Dimca, Singularities and topology of hypersurfaces, Universitext, SpringerVerlag, New York, 1992. MR1194180 (94b:32058)

[H] June Huh, The maximum likelihood degree of a very affine variety, Compos. Math. 149 (2013), no. 8, 1245-1266, DOI 10.1112/S0010437X13007057. MR3103064

[HS] June Huh and Bernd Sturmfels, Likelihood geometry, Combinatorial algebraic geometry, Lecture Notes in Math., vol. 2108, Springer, Cham, 2014, pp. 63-117, DOI 10.1007/9783-319-04870-3_3. MR3329087

[LL] T. Y. Lam and K. H. Leung, On vanishing sums of roots of unity, J. Algebra 224 (2000), no. 1, 91-109, DOI 10.1006/jabr.1999.8089. MR.1736695(2001f:11135)

[RW] J. Rodriguez and B. Wang, The maximum likelihood degree of rank 2 matrices via Euler characteristics, arXiv:1505.06536

Department of Mathematics, University of Wisconsin-Madison, Madison, Wisconsin 53706

E-mail address: wang@math.wisc.edu 